\title{
Mass Distribution as an Approach for Designing Lightweight-Driven Product Architectures
}

\author{
Felix Laufer ${ }^{1}$, Daniel Roth ${ }^{1}$, Hansgeorg Binz ${ }^{1}$ \\ ${ }^{1}$ University of Stuttgart \\ felix.laufer@iktd.uni-stuttgart.de \\ daniel.roth@iktd.uni-stuttgart.de \\ hansgeorg.binz@iktd.uni-stuttgart.de
}

\begin{abstract}
Lightweight-design methods for the conceptual phase of the product-development process are becoming increasingly important due to their influence on products' mass. Besides mass reduction, the redistribution of mass within the product can support the designer in addressing lightweight-design motivations more effectively. This paper introduces a method of systematic mass distribution using the design structure matrix (DSM). Various motivations for lightweight design are therefore transformed into dependencies among product components. A case study of a high-pressure water-jetting unit serves as an application example to illustrate the procedure introduced. Based on a clustering algorithm, novel lightweight-driven product architectures are derived to support optimized mass distribution for the jetting unit.
\end{abstract}

Keywords: lightweight design, mass distribution, product architecture, DSM

\section{Introduction}

When focusing on lightweight design, the emphasis is usually on reducing the mass of a product. The motivations for doing so are manifold: Reducing mass for saving energy during use, reducing mass for reducing manufacturing costs, or reducing mass for improving the working performance of a dynamic product are just a few examples. However, some of these motivations for performing lightweight design are not only achievable by reducing mass, but also by redistributing mass. Let us consider lowering the center of gravity (c.g.) of a car for better driving characteristics as the motivation for lightweight design. Reducing the mass of the car roof satisfies the motivation, as does lowering the engine by a few inches within the chassis. This repositioning of product parts - which is a redistribution of mass - is often accompanied by a change in the product architecture (PA) because mass is tied to physical product components. While lowering the car's engine is a relatively small change, larger changes in the PA require methodological support, especially when it comes to rather complex products (Lindemann et al., 2009). Both lightweight design and the design of the PA are important tasks during the conceptual phase of the product-development process (Mayyas et al., 2018; Richter et al., 2016) and can (with regard to the redistribution of mass) be linked to each other. This 
linkage enables PA methods to be transferred to conceptual lightweight design by way of support for designing lightweight-driven PAs through mass distribution (Laufer et al., 2019).

\section{Problem Statement, Goal, and Structure}

Due to the design freedom offered by the conceptual phase of the product-development process, the designer is able to shape the PA of a product. Two general possibilities arise when designing the PA: Integration (preferred in lightweight design) and modularization, which are often referred to as "conflicting requirements" (Richter et al., 2016). Researchers have particularly demonstrated that designs that are strongly driven by technical constraints (such as mass with its quantity and distribution) tend to exhibit a more integral PA than those that do not have these constraints (Hölttä-Otto \& de Weck, 2007). However, an integrated PA alone is not sufficient to satisfy all relevant motivations for a specific lightweight-design case. Usually, all the relevant motivations are taken into account once the basic architecture has already been set. Gumpinger (2016), for instance, presents a framework for modular lightweight design, where lightweight motivations are satisfied by optimizing an already developed PA. The problem occurring with such an approach is that potentials for lightweight design within the architecture of a product (which cannot be realized through integration alone) are not exploited. To refer to the example in the introduction, the c.g. of a car can even be lifted if an integrated (i.e., light) design is realized in the lower chassis area. Lightweight design therefore needs to be aware of lightweight potentials within the PA early in the conceptual phase of product development. To realize these potentials, the authors found that the link between the two fields "lightweight design" and "PA design" lies in the distribution of mass. Mass is tied to the physical components of a product. The way in which these components are structured is represented in the product structure that forms part of the PA. A guided mass distribution (i.e., (re)positioning of components) can thus meet motivations for lightweight design with the help of PA design methods during the conceptual phase (Laufer et al., 2019). This subsequently leads into the question of which PA methods are suitable for lightweight design with a focus on the distribution of components' mass. Here, the authors found that matrix-based methods, especially the design structure matrix (DSM), are suitable for designing a PA with regard to lightweight design through mass distribution (Laufer et al., 2019). This result leads to the research question for this paper, formulated as follows: How can the DSM be applied to mass redistribution with the aim of deriving lightweight-driven product architectures? This paper therefore aims to operationalize the DSM for the derivation of lightweight-driven PAs.

Consequently, Section 3 provides an overview of the mass distribution with relevance for lightweight design in the conceptual phase and summarizes current DSM applications in lightweight design. Section 4 introduces a methodological procedure for deriving a lightweightdriven PA using an application example of a high-pressure water-jetting unit. A critical discussion of the results is given in Section 5 before the paper is concluded in Section 6.

\section{State of the Art}

This section provides an overview of two topics: Firstly, a literature perspective on lightweight design with a focus on mass distribution is given and, secondly, basics of the DSM are generally discussed with a particular emphasis on application to lightweight design.

\subsection{Mass Distribution in Lightweight Design}

When studying mass distribution in lightweight design, airplane and race-car design are highly relevant fields due to the sensitive mass limits and the stringent requirements regarding flight 
and driving characteristics, respectively. In airplane design, the mass limits are set at the beginning of the conceptual phase after the mass of every section has been determined from previous models. Here, high accuracy of the initial mass prediction is a prerequisite. By setting these mass goals, the various design departments (fuselage, wings, etc.) are given the mass figures that serve as a springboard for further evaluation. The overall goal is to ensure that the mass distribution meets the acceptable fore-and-aft limits of the location of the center of gravity. A precise c.g. location is imperative for an airplane's flight stability (Torenbeek, 1976).

The location of the c.g. is equally important when designing a race car, whereby mass is distributed to ensure a c.g. as close as possible to the ground to enable high cornering speeds. For that purpose, race-car designers undershoot the minimum weight limit mandatorily required by regulations to further lower the c.g. with extra ballast weights. Additionally, large masses (like the engine) are positioned close to the yaw axis in order to minimize the mass moment of inertia with respect to this axis. This improves the car's agility (Trzesniowski, 2014).

Both the center of gravity and mass moment of inertia are also important parameters in robotic design, where lightweight components are enablers of high performance. Borchert et al. (2015) present an investigation into the positioning of three motors for a delta robot. The relatively high mass of the motors influences the dynamic characteristics of the robot, thus the mass distribution constitutes a significant factor for the robot's performance metrics. When it comes to bionically inspired walking robots, the distribution of mass is considered early in the conceptual-design phase to realize a robot concept with high stability during motion (Wojtkowiak et al., 2017).

In medical engineering, the design of lightweight prostheses for patients with below-knee amputations is sensitive to the distribution of mass due to issues of gait efficacy (Lehmann et al., 1998). A proximal center of mass produces a more efficient gait which (alongside the distribution of mass) leads to the use of lightweight materials for distal components (e.g., feet). Moreover, the compactness of lightweight components increases the comfort and usability of prostheses (Ahn et al., 2017), which might also be realized through a redistribution of mass.

Ultimately, the distribution of mass can facilitate an integrated design. If two components with high functional interaction are spatially separated, the redistribution of these components close to each other makes integrated design feasible. Pimmler \& Eppinger (1994) demonstrate the rearrangement of components in a climate-control system to realize a more integrated design within the redesigned modules.

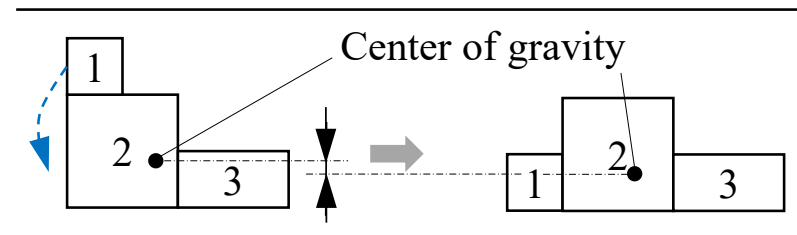

Optimization of the c.g. location

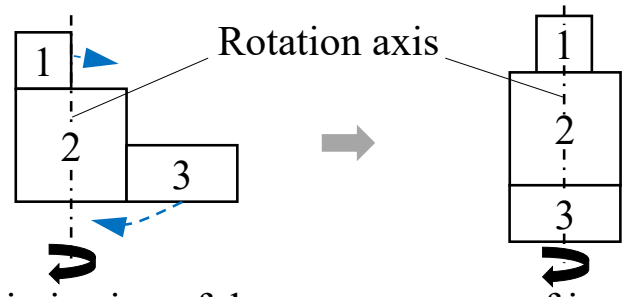

Optimization of the mass moment of inertia

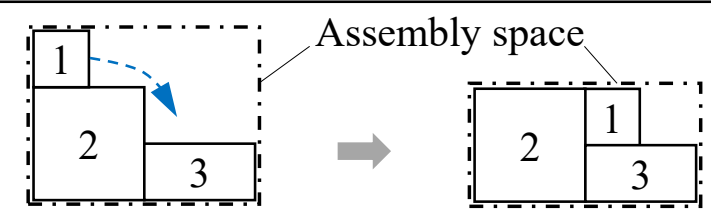

Increase in compactness

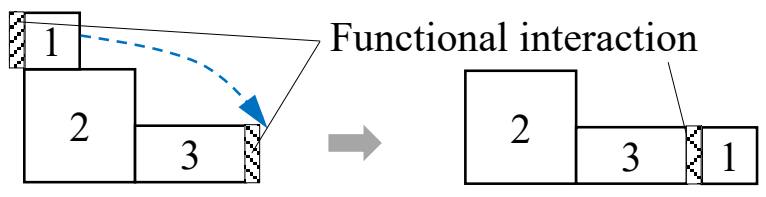

Enabling an integrated design

Figure 1. Options for mass redistribution within a product to satisfy lightweight-design motivations (modified from Laufer et al. (2019))

To summarize the perspectives from the relevant literature, several motivations for lightweight design can be satisfied through mass distribution. The authors published an overview of the 
most relevant motivations for lightweight design by varying a three-part object (indicated by 1 , 2, and 3, see Figure 1), the mass of which was systematically redistributed to satisfy these motivations (Laufer et al., 2019).

\subsection{Application of DSM in Lightweight Design}

The design structure matrix dates back to Steward (1981), when it was applied to analyze the structure of a system's design. The DSM is a square matrix that shows the dependencies between elements in a visually and analytically advantageous manner (Browning, 2001). These elements might be physical product components or process tasks, for instance, and the DSM is often used to depict and structure information in complex systems (Lindemann et al., 2009). After entering all the interactions into the DSM (analysis), clustering forms the elements into system chunks (synthesis). Here, the cluster algorithms aim to find the maximally interacting chunks by rearranging the rows and columns of the DSM. As such, these chunks are clusters of elements that are interconnected to a high degree while being relatively disconnected from the rest of the system (Lindemann et al., 2009).

Since the DSM method targets modularization-although the module-inherent dependencies are high, thus revealing options for integration - the application to explicit lightweight-design scenarios is not broadly exploited. Luft et al. (2014) use the DSM for a matrix-based product description as a starting point for mass and cost optimization. The focus of the approach is on the overview of a complex thermal management system to handle the various dependencies before re-structuring the architecture. From a requirements-management perspective, McLellan et al. (2009) apply the DSM and domain-mapping matrices (DMM) to prioritize requirements that significantly influence mass reduction. By mathematically manipulating the DMM (rearrangement of requirements to components), varying performance concerns could be eliminated among different designers while conducting lightweight design. Wang et al. (2018) combine axiomatic design with the DSM to develop a functionally integrated design by mapping functional requirements and various design parameters. A case study presents an integrated concept for a suspension design with an integrated electric drive.

To summarize the view from literature, the use of DSMs for application in lightweight design is limited on either requirements engineering or the integration of functions or components. No prescriptive approach is yet available that applies DSM to the guided distribution of mass to meet various lightweight motivations.

\section{Applying the DSM to Derive Lightweight-Driven PAs: Case Study of a High-Pressure Water-Jetting Unit}

This section introduces a method for guided mass distribution to satisfy lightweight-design motivations using the DSM. An application example is used to illustrate the methodological procedure. This section is divided into the following four subsections: Firstly, the requirements for the method are defined, and the required outcome is determined. The procedure model from which a lightweight-driven PA can be derived is introduced. Secondly, an application example of a high-pressure water-jetting unit is introduced, and the product is broken down into its constituent assemblies (decomposition). Thirdly, the various lightweight motivations summarized in Figure 1 are systematically interpreted as dependencies among system elements to make them applicable for the DSM. The application example is employed to illustrate this procedure by analyzing the assembly dependencies. Lastly, the synthesis step of the method is derived before being applied to the high-pressure water-jetting unit. 


\subsection{Requirements for the Method and Procedural Model}

The method to be developed must meet several requirements while analyzing mass distribution for lightweight design. On the one hand, these requirements originate from a lightweight context as the framework for this paper. On the other hand, requirements arise when applying methods for handling complex systems because the DSM falls into that category (Steward, 1981). Additionally, the corresponding meta-requirements for methods in general according to Keller et al. (2009) are assigned to the requirements listed in parentheses.

From a lightweight-design perspective, the method must be able to address the lightweight motivations from a mass-distribution standpoint as presented in Section 3.1 (compatibility). Furthermore, it must be applicable to various products and not be predetermined to a product category or industry (flexibility). As the various motivations are of differing importance within various lightweight projects, the method must provide a simple weighting of these motivations against each other.

In the context of managing complex systems, the application of the method must be justified by a certain complexity of the technical system at hand (usefulness). In the case of an undercomplex system, the efficiency of an application using the DSM would not be accepted by the user (Lindemann et al., 2009). Lastly, while operating on an abstract level for both the analysis and synthesis of technical systems, the results must be transparent and repeatable when applied by different users with the same level of product knowledge (comprehensibility and repeatability).

Eventually, the procedure model of the developed method is introduced, as illustrated in Figure 2. The inherent logic of the model follows the three consecutive steps of decomposition, analysis and synthesis. This structure and the presented sub-steps are methodologically derived in the following subsections (4.2 to 4.4).

\begin{tabular}{|c|c|c|}
\hline $\begin{array}{l}\text { Decomposition } \\
\text { (cf. Section 4.2) } \\
\text { - Identify system } \\
\text { elements' level of } \\
\text { granularity } \\
\text { - Decompose system } \\
\text { into suitable elements } \\
\text { - Reduce all elements } \\
\text { to mass-relevant } \\
\text { elements }\end{array}$ & $\begin{array}{l}\text { Analysis (cf. Section 4.3) } \\
\text { - Choose lightweight- } \\
\text { relevant motivations and } \\
\text { weight them (in \%) } \\
\text { - Determine interactions } \\
\text { between elements for all } \\
\text { chosen motivations and } \\
\text { score them ( } 0 \text { to } 4 \text { ) } \\
\text { - Combine all matrices into } \\
\text { one using weightings and } \\
\text { individual cell entries }\end{array}$ & $\begin{array}{l}\text { Synthesis (cf. Section 4.4) } \\
\text { - Choose threshold values } \\
\text { - Derive binary DSMs } \\
\text { - Apply clustering algorithm } \\
\text { - Derive lightweight-driven } \\
\text { modules within the DSMs } \\
\text { - Compare the various } \\
\text { product architectures } \\
\text { - Design first basic } \\
\text { lightweight concepts }\end{array}$ \\
\hline
\end{tabular}

Figure 2. Procedure model for the derivation of lightweight-driven PAs

\subsection{Decomposition: Application Example of a High-Pressure Water-Jetting Unit}

To illustrate the methodological steps of the procedure introduced, an application for the authors' industrial partner WOMA Ltd. will serve as an evaluation example throughout this paper. A mobile, high-pressure water-jetting unit is therefore investigated with regard to deriving a more lightweight-driven PA. The pressure unit is illustrated in Figure 3. Faced with market challenges like elevated performance requirements, the product has become heavier over the past product generations. This conflicts with the upper mass limits of the customers' requirements. Moreover, due to an increase in the products' complexity that has simultaneously grown with the overall architecture, the company decided to employ a fundamental revision of the PA of the product, thereby redesigning the entire concept. Lightweight-design 
considerations are declared to be the focus of this new PA concept. As such, lightweight motivations achievable through mass redistribution were screened (see Figure 1). The results revealed scores for "enabling integrated design" (weighted at $50 \%$ ), "increasing the compactness" (weighted at $40 \%$ ) and "lowering the c.g." (weighted at $10 \%$ ) to be the most important motivations in this particular lightweight-design project. The weighting was decided in conjunction with the industrial partner and is mentioned in this section due to the better linkage to the actual motivations selected.

Since the DSM uses information about a product, the design progress corresponds with the information available. For a lightweight-driven analysis, information on mass and geometry is required. This information can be estimated (see Posner, 2016) or taken from previous model generations. Due to the fact that the product belongs to a product generation that has grown over time, current product parts can be used as input for analysis in this particular case. Furthermore, the granularity of the decomposition is set on an assembly level. This seems to be the most feasible perspective for redesigning the entire PA. A decomposition on a component level appears too fine, thus adding unnecessary complexity to the analysis and synthesis. However, this might be a reasonable next step for later considerations in the design process.

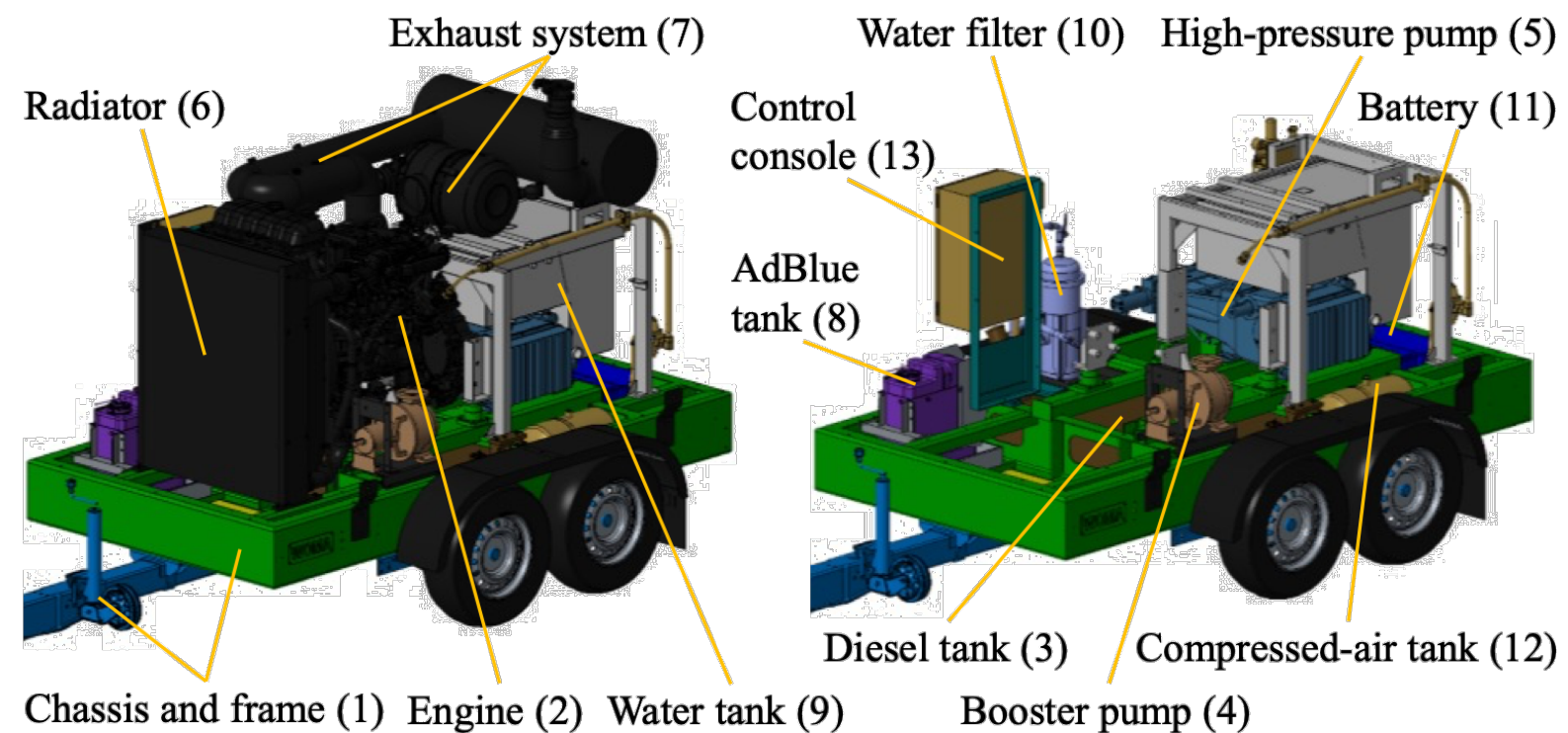

Figure 3. Isometric view of the high-pressure water-jetting unit

The main function of the jetting unit is to produce a water jet at a high pressure of up to 3,000 bar. The decomposition is performed by analyzing this function using its active assemblies, which then represent the mass-relevant elements. The water jet can be used for cleaning or cutting processes and is mounted on a chassis and frame (1) for mobility purposes. The main function is split into two separate paths: On the one hand, an engine (2) combusts diesel from a diesel tank (3) (embedded in the frame) to produce rotational energy. This energy is primarily used to power the booster pump (4) and the high-pressure pump (5). Furthermore, the engine is cooled by a radiator (6). The exhaust gases are filtered through an exhaust system (7) on top of the engine and additionally post-treated using AdBlue (8) additive (diesel path). On the other hand, water is stored in a water tank (9), then pumped by a booster pump (up to 6 bar) into a water filter (10). Downstream of the filter, the water enters the high-pressure pump where the pressure is increased to 3,000 bar (water path). The rear of the frame houses the battery (11) and compressed-air tank (12) used for switching the valves of the high-pressure pump. A control console (13) is placed on the side at standing height for an ergonomic operating position. For protection purposes, the entire product is covered by a hood that sits on the frame. This hood is not an object of investigation in this paper and is thus not displayed in Figure 3. 


\subsection{Analysis: Motivations for Lightweight Design as Dependencies Between Different Product Components}

The application of the binary DSM builds upon dependencies between elements. Pimmler \& Eppinger (1994) define the dependencies between components as interactions on a functional level. They develop a scheme for systematically identifying and describing the interactions in four categories: special alignment, energy exchange, information exchange, and material exchange between components. These categories (except "special alignment") are used within this paper to delineate the lightweight motivation "enabling integrated design" (see Figure 1, lower right-hand corner). Integrated design is thus interpreted as the physical proximity of components that have a functional dependency (i.e., interaction on a material, energy, or information level). For instance, a fuel hose between an engine and a fuel tank leads to an interaction between the engine and tank on a material-exchange level (fuel is fed from the tank to the engine). As Pimmler \& Eppinger (1994) explain, the DSM can be used to record this interaction by making an entry in the corresponding matrix cell. Furthermore, the lightweight context of this paper requires a distinction between the various interaction types because the influence on lightweight design (i.e., the influence on mass) might differ from one interaction to another. The interaction of transmitting torque through a shaft, for instance, might have more mass to it than the interaction of transmitting electricity through a cable. In this particular case, a guided mass distribution has to favor the spatial proximity of two components transmitting torque through a shaft than of two components transmitting energy through a cable. Within the paper's application example, when the engine transmits torque through a shaft to the highpressure pump, these two assemblies should be positioned close to each other, otherwise the shaft will need to be longer, thereby adding more mass to the system. When the battery transmits electricity to the engine, spatial separation would also be disadvantageous for mass considerations. However, the mass of the cable is less than the shaft, and therefore also less important for mass considerations. Consequently, weighting is performed by assigning points from 0 (where there is no interaction) to 4 (for interaction with high functional mass relevance), see Figure 4 (i.e., engine/high-pressure pump $=4$; engine/battery $=1$ ). Note that the qualitative equivalent for the different points must be adapted to each individual design case depending on the relative functional exchanges. The frame carrying all assemblies will be an object of investigation for a topology optimization in a later project. A firm functional dependency within the paper's context is not given (see "no frame interaction" in Figure 4).

\begin{tabular}{|c|c|c|c|c|c|c|c|c|c|c|c|c|c|c|}
\hline & & 1 & 2 & 3 & 4 & 5 & 6 & 7 & 8 & 9 & 10 & 11 & 12 & 13 \\
\hline Frame & 1 & & & & & & & & & & & & & \\
\hline Engine & 2 & & & 1 & 3 & 4 & 3 & 3 & & & & 1 & & 1 \\
\hline Diesel tank & 3 & & 1 & & & & & & & & & & & \\
\hline Booster pump & 4 & & 3 & & & 2 & & & & 1 & 2 & & & 1 \\
\hline High-pressure pump & 5 & & 4 & & 2 & & & & & & 3 & & 2 & 1 \\
\hline Radiator & 6 & & 3 & & & & & & & & & & & \\
\hline Exhaust system & 7 & & 3 & & & & & & 1 & & & & & \\
\hline AdBlue tank & 8 & & & & & & & 1 & & & & & & \\
\hline Water tank & 9 & & & & 1 & & & & & & & & & \\
\hline Water filter & 10 & & & & 2 & 3 & & & & & & & & \\
\hline Battery & 11 & & 1 & & & & & & & & & & & 1 \\
\hline Compressed-air tank & 12 & & & & & 2 & & & & & & & & 1 \\
\hline Control console & 13 & & 1 & & 1 & 1 & & & & & & 1 & 1 & \\
\hline & 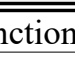 & I-TIC & $\overline{\mathrm{rel}}$ & ance & & & & & & & $\mathrm{Hi}$ & & Very & high \\
\hline & & & & oints & & & & & & & & & & \\
\hline
\end{tabular}


Figure 4. DSM for functional interaction with corresponding mass-dependency scheme (cells with a value of " 0 " have been left empty)

The next lightweight motivation to be interpreted as a dependency between components - to facilitate usage in the DSM - is the "increase of the compactness." As depicted in Figure 1 (lower left-hand corner), the occupied assembly space must be reduced in order to increase the compactness. This is carried out by taking a product component and repositioning it into a vacant, available product space. However, a distinction must be made between the increase in compactness and the integration of components' functions. According to Roth (2000), the integration of functions or components requires a merger of at least two working surfaces or two working spaces (e.g., merging two component walls). By contrast, the repositioning of a component into a vacant space only constitutes "embedding" without any kind of merger. This fact distinguishes the motivation "increase of the compactness" from "enabling an integrated design" (which, in contrast, can lead to an integration of functions or components). Within the application example, the high-pressure water-jetting unit's frame possesses enough space to embed the battery inside its structure, thus the compactness can be increased without performing any integration. This increase in compactness, however, is individual for every product, as well as limited by many boundary conditions, especially geometric ones. These geometric conditions might enable embedding between some components and can thus be interpreted as geometric dependencies. Lindemann et al. (2009) also use the DSM to record geometric dependencies between product parts. In order to pick up on this aspect in this paper's lightweight context, the mass-relevant aspect of compactness manifests itself in the space occupied by the product components, thus weighting within the DSM displays the different assembly spaces of components that are able to be embedded in other ones. Figure 5 illustrates this dependency based on the application example. Here, the frame constitutes the assembly with the most space for other assemblies to be embedded. The diesel tank, the battery, and the AdBlue tank are already inserted into the frame (see Figure 3). However, the frame provides more free space for a further increase in compactness. When it comes to weighting, 4 points are assigned to embeddable assemblies with very large relative assembly space, while 0 points are assigned to instances where there is no possibility for embedding between assemblies, see Figure 5. For instance, the diesel tank that is larger in size is weighted more highly than the AdBlue tank as an assembly that can be embedded into the frame (i.e., diesel tank/frame $=4$; AdBlue tank/frame $=2$ ). Note that the qualitative equivalent for the various points must be adapted to each individual design case based on the relative assembly space.

\begin{tabular}{|c|c|c|c|c|c|c|c|c|c|c|c|c|c|c|}
\hline & & 1 & 2 & 3 & 4 & 5 & 6 & 7 & 8 & 9 & 10 & 11 & 12 & 13 \\
\hline Frame & 1 & & & 4 & 2 & & & & 2 & 4 & 2 & 2 & 3 & \\
\hline Engine & 2 & & & & & & & & & & & & & \\
\hline Diesel tank & 3 & 4 & & & & & & & & & & & & \\
\hline Booster pump & 4 & 2 & & & & & & & & & & & & \\
\hline High-pressure pump & 5 & & & & & & & & & & & & & \\
\hline Radiator & 6 & & & & & & & & & & & & & \\
\hline Exhaust system & 7 & & & & & & & & & & & & & \\
\hline AdBlue tank & 8 & 2 & & & & & & & & & & & & \\
\hline Water tank & 9 & 4 & & & & & & & & & 2 & & & \\
\hline Water filter & 10 & 2 & & & & & & & & 2 & & & & \\
\hline Battery & 11 & 2 & & & & & & & & & & & & \\
\hline Compressed-air tank & 12 & 3 & & & & & & & & & & & & \\
\hline Control console & 13 & & & & & & & & & & & & & \\
\hline Emb & ddab & as & bbly & pace & & & & & & & & & Ver & $\operatorname{large}$ \\
\hline & & & & ints & 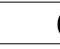 & & & & & & & & & \\
\hline
\end{tabular}


Figure 5. DSM for increase in compactness with corresponding mass-dependency scheme (cells with a value of " 0 " have been left empty)

Finally, a distribution of mass to fulfill the "optimization of the c.g. location" and the "optimization of mass moment of inertia" are to be discussed. These two motivations are dealt with in the same way due to one major consideration: Both motivations are driven by mass accumulations in terms of to the various axes of a technical system. While the optimal location of the c.g. along the gravitational axis requires as much mass as possible towards the desired extremum, the optimization of the mass moment of inertia requires either a maximum mass accumulation on the rotational axis (low mass moment of inertia) or as far away as possible from the rotational axis (high mass moment of inertia). These "high-mass areas" cannot be directly represented by dependencies between components. Initially, the assumption must be made that modules with a high mass (accumulated by the heaviest components within the product) enable designers to design for an optimized c.g. location and an optimized mass moment of inertia, respectively. Thereby, a correlation can be made with respect to the DSM nomenclature, which records a "dependency" (in this case, rather a "connection") between components with high masses. To establish this connection, the masses of the various components in relation to the entire product first need to be weighted. This weighting is conducted by means of an $\mathrm{ABC}$ analysis. While A parts (most mass-intensive components; e.g., engine) score 4 points, $C$ parts (negligible mass; e.g., battery) score 0 points. The connection between two components then defines the mean value between the weighting. For instance, the engine's mass scores 4 points, and a water tank's mass scores 2 points, while the connecting cell in the DSM is set at $3(=(4+2) / 2)$, as shown in Figure 6 . Additionally, if a C part $(=0$ points) is correlated to any other component, the connecting cell is set at 0. Otherwise, lowmass components would be connected to components with a high mass, thus diluting the creation of high-mass modules.

\begin{tabular}{|r|c|c|c|c|c|c|c|c|c|c|c|c|c|c|c|}
\hline Individual points & & 1 & 2 & 3 & 4 & 5 & 6 & 7 & 8 & 9 & 10 & 11 & 12 & 13 \\
\hline Frame & 3 & 1 & & 3.5 & 3 & & 3 & & & & 2.5 & & & & \\
\hline Engine & 4 & 2 & 3.5 & & 3.5 & & 3.5 & & & & 3 & & & & \\
\hline Diesel tank & 3 & 3 & 3 & 3.5 & & & 3 & & & & 2.5 & & & & \\
\hline Booster pump & 0 & 4 & & & & & & & & & & & & & \\
\hline High-pressure pump & 3 & 5 & 3 & 3.5 & 3 & & & & & & 2.5 & & & & \\
\hline Radiator & 0 & 6 & & & & & & & & & & & & & \\
\hline Exhaust system & 0 & 7 & & & & & & & & & & & & & \\
\hline AdBlue tank & 0 & 8 & & & & & & & & & & & & & \\
\hline Water tank & 2 & 9 & 2.5 & 3 & 2.5 & & 2.5 & & & & & & & & \\
\hline Water filter & 0 & 10 & & & & & & & & & & & & & \\
\hline Battery & 0 & 11 & & & & & & & & & & & & & \\
\hline Compressed-air tank & 0 & 12 & & & & & & & & & & & \\
\hline Control console & 0 & 13 & \multicolumn{8}{|c|}{ Relative assembly's mass } & Negligible & Low & Medium & High & Very high \\
\hline \hline
\end{tabular}

Figure 6. DSM for lowering the c.g. through mass-intensive modules with a corresponding mass-dependency scheme (cells with a value of " 0 " have been left empty)

Subsequently, all relevant motivations for lightweight design (see Figure 1) can be represented as dependencies between product elements (i.e., assemblies). In other words, lightweight motivations are expressed as the mass-relevant attributes of - and amongst - components, while components and their interactions constitute the product structure (see Figure 7). As the 
product structure forms part of the PA (Richter et al., 2016), this ultimately allows lightweightdriven PAs to be derived.

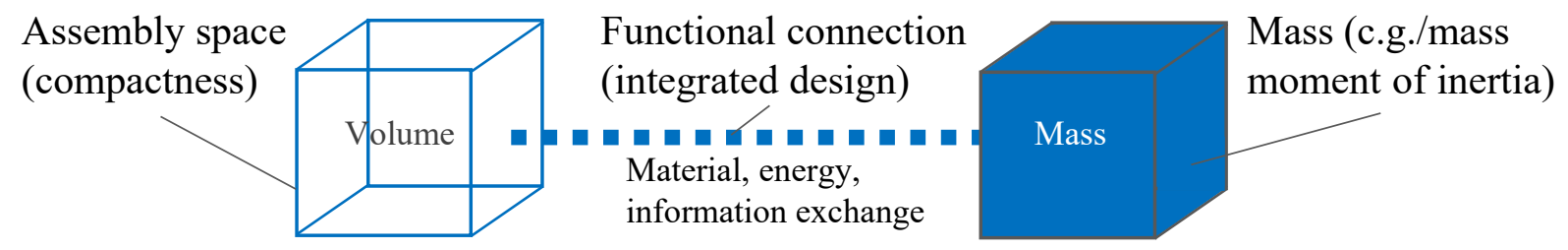

Figure 7. Three essential properties of the product structure from a lightweight perspective

Finally, after making it possible for the various lightweight motivations to be implemented in the DSM, the next step is to combine these motivations within the DSM. Multi-information cell entries have previously been investigated by other researchers. Pimmler \& Eppinger (1994) use multi-entry cells in the DSM to combine information on the material, energy, information, and spatial interactions of a climate-control system, as explained earlier on. However, after defining the material interaction as the most important interaction type, the clustering algorithm focuses exclusively on this interaction. Lindemann et al. (2009) showcase a PA study of a high-pressure pump deriving three different DSMs. These different "views" of the product (functional, geometric, feature-based) were also classified by importance. Likewise, the most important dependency (i.e., functional) was clustered and formed the framework for the other two dependency categories that were then superposed. Nevertheless, the possibility of weighting each perspective and combining the various aspects of dependencies into one single DSM before clustering to form modules is identified as feasible by Pimmler \& Eppinger (1994). This approach is chosen for the methodological procedure within this paper for two reasons: Firstly, by choosing one interaction perspective over all others, the process of differentiation is binary. Weighting compares the different perspectives relatively and enables a more integrated display of all relevant lightweight motivations. However, weighting might differ between one group of components and another that combines disparate preferences. This might blur the clustering results. Secondly, clustering three DSMs (integrated design, compactness, lowering the c.g.) separately and subsequently superposing them is significantly more time-consuming than clustering one DSM. Since the handling of complex systems is generally a time-consuming process, the authors are convinced of deriving expedient results with this approach while executing the analysis more efficiently. One main drawback might be reduced transparency throughout the clustering process with regard to the results. Figure 8 thus shows the combined DSM. The accumulated cell entry is calculated as $\sum_{1}^{3} w_{i} * j_{i}$, where $w_{i}$ is the corresponding weight and $j_{i}$ the individual cell entry, see Figures $4-6$ (e.g., the entry for the diesel tank/frame cell is calculated as follows: $0.5 * 0+0.4 * 4+0.1 * 3=1.9)$. 


\begin{tabular}{|r|c|c|c|c|c|c|c|c|c|c|c|c|c|c|}
\hline & 1 & 2 & 3 & 4 & 5 & 6 & 7 & 8 & 9 & 10 & 11 & 12 & 13 \\
\hline Frame & 1 & & 0.4 & 1.9 & 0.8 & 0.3 & & & 0.8 & 1.9 & 0.8 & 0.8 & 1.2 & \\
\hline Engine & 2 & 0.4 & & 0.9 & 1.5 & 2.4 & 1.5 & 1.5 & & 0.3 & & 0.5 & & 0.5 \\
\hline Diesel tank & 3 & 1.9 & 0.9 & & & 0.3 & & & & 0.3 & & & & \\
\hline Booster pump & 4 & 0.8 & 1.5 & & & 1.0 & & & & 0.5 & 1.0 & & & 0.5 \\
\hline High-pressure pump & 5 & 0.3 & 2.4 & 0.3 & 1.0 & & & & & 0.3 & 1.5 & & 1.0 & 0.5 \\
\hline Radiator & 6 & & 1.5 & & & & & & & & & & & \\
\hline Exhaust system & 7 & & 1.5 & & & & & & 0.5 & & & & & \\
\hline AdBlue tank & 8 & 0.8 & & & & & & 0.5 & & & & & & \\
\hline Water tank & 9 & 1.9 & 0.3 & 0.3 & 0.5 & 0.3 & & & & & 0.8 & & & \\
\hline Water filter & 10 & 0.8 & & & 1.0 & 1.5 & & & & 0.8 & & & & \\
\hline Battery & 11 & 0.8 & 0.5 & & & & & & & & & & & 0.5 \\
\hline Compressed-air tank & 12 & 1.2 & & & & 1.0 & & & & & & & & 0.5 \\
\hline Control console & 13 & & 0.5 & & 0.5 & 0.5 & & & & & & 0.5 & 0.5 & \\
\hline
\end{tabular}

Figure 8. Combined DSM with weighted entries (cells with a value of "0" have been left empty)

\subsection{Synthesis: Deriving Novel Lightweight-Driven PAs}

After weighting the various motivations and combining them into one single DSM, the analysis is completed, and the synthesis starts. Due to the fact that the DSM cells contain different numbers (ranging from 0 to 4 in decimal increments due to the combination of different entries and weights, see Figure 8), two general clustering procedures are possible: Either a numerical DSM that is able to cluster decimal-cell entries can be used or, alternatively, a threshold value can be set. This filters all entries below a certain value and sets the remainder to 1, thereby generating a binary DSM. For the paper's methodological procedure (see Figure 2), the authors have opted for an explicit threshold value. Initial experiments have revealed a range for the threshold value in which comprehensible clustering results (range from approx. 0.8 to 1.2) were produced. The benefit of a threshold value lies in the generation of several product architectures that can be compared with each other. Furthermore, by setting the threshold relatively high (range from approx. 1.0 to 1.5), so called "core modules" can be derived that represent the strongest lightweight-driven dependencies. However, this threshold might also blur the results by overloading the user with excessive information due to the multiple reorganization steps, thereby undermining the initial idea of making the result more effective. Further work will use a numerical DSM for clustering and compare the different approaches. Nevertheless, since the abstract presentation of system modules in clusters with binary entries ( 0 or 1$)$ is often difficult to use as a reliable basis for further conceptual designs, the comparison of various clustering results might enable a more differentiated perspective on novel lightweight-driven architectures. For clustering within this paper, a heuristic swapping algorithm is used within an Excel spreadsheet with specialized macros. Ultimately, Figure 9 depicts two clustered DSMs and core modules (frame and engine pumps) for threshold values 1.0 and 1.2. 

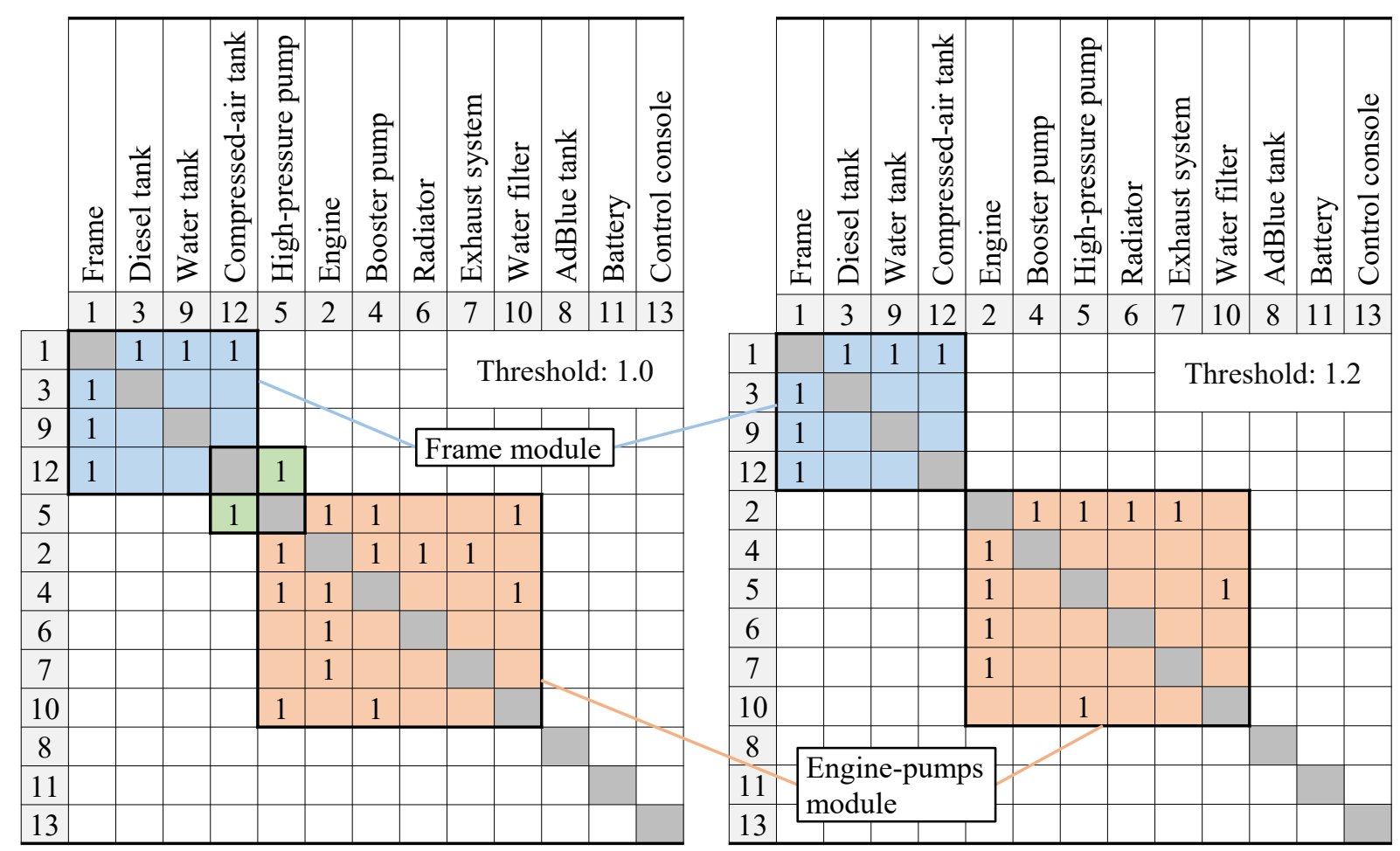

Figure 9. Clustered DSMs with formed (frame and engine-pumps) core modules for two different threshold values (cells with a value of "0" have been left empty)

While a threshold value of 0.8 (not shown) did not deliver significant, modularizable results, both DSMs depicted show clear module formation. Comparing these results with each other and with the initial product, several aspects are important with regard to lightweight-driven PA design:

- The blue-colored core module for the frame in the upper left-hand corner that appears identically for both thresholds consists of the following assemblies: frame, diesel tank, water tank, and compressed-air tank. With the exception of the water tank, the initial concept comprises this module. However, the synthesis shows that the water tank is preferably to be installed in the frame due to its high mass in order to lower the c.g. and, more importantly, to significantly reduce assembly space in the rear part of the highpressure water-jetting unit. This might affect service issues of the water tank that can be resolved by making it accessible from the outer frame.

- The red-colored "engine-pumps" core module in the lower right-hand corner consists of the engine, both pumps, radiator, exhaust system, and water filter in both DSMs. While the "Threshold: 1.2" matrix displays the engine as centrally interconnected to the other assemblies, the "Threshold: 1.0" matrix illustrates the high-pressure pump as more central due to the compressed-air interaction (green-colored module) that links both large modules (red- and blue-colored). In the current design, there is already a close arrangement of the following assemblies: engine, booster pump, and high-pressure pump. However, the water filter located on the other side of the unit (see Figure 3) appears to be unnecessarily extending the water path, thus adding mass to the system. The radiator and exhaust system are revealed to be appropriately located within the system.

- The assemblies that appear out of interaction are the AdBlue tank, the battery and the control console. These system elements have a low-rated functional dependency as well as a relatively small assembly space (see Figure 4 and 5) that let them drop below the threshold value. This observation indicates that these components (especially the AdBlue tank and battery) might be easily embeddable in the frame but are less important 
than, for instance, the water tank to be embedded. This finding might lead to a design prioritization when it comes to a redesign of a lightweight-driven PA.

\section{Discussion}

The clustering results revealed a preferred lightweight-driven modularization that is close to the current PA of the high-pressure water-jetting unit. However, several optimization suggestions could be derived using the method introduced. One reason for the rather incremental improvement of the design might be the geometrical focus of the method. In particular, the step for compactness-dependency implementations in the DSM can predetermine the designer's view of radical concepts because it builds upon existing assemblies' geometries. Nevertheless, the analysis and synthesis showed significant potential for a redistribution of the water tank's mass into the frame, which would lead to a more lightweight-driven PA. Furthermore, the method does not support the designer in the module-inherent organization of the assemblies, although this is fundamental for designing for the c.g. location and the mass moment of inertia. This will be a next step in the methodological development process.

By focusing solely on lightweight design, other PA-relevant issues might fall short within the design process. When discussing the results with a team of four designers (2-3 years of practical knowledge, methodological experts, no prior knowledge of application example), the foremost maintenance issues regarding integrated components were discussed from a design perspective. Assemblies either embedded or integrated inside the frame, for instance, appear to be critical when maintenance intervals are very frequent. To tackle this problem, an extension of the method might consider module drivers as discussed by Ericsson \& Erixon (1999) to populate other important module motivations alongside lightweight ones.

The next step is to support designers from WOMA Ltd. in translating the rather abstract synthesis results into an actual conceptual design. This will involve many trade-off decisions (e.g., embedding or even integrating the water filter into the water tank (see Figure 4-5) versus a more complicated maintenance routine) that must be addressed individually.

The last step is to evaluate the requirements for the method from Section 4.1. Due to the fact that these motivations were transformed into the DSM nomenclature, compatibility is satisfied. Concerning the flexibility of the method, other products must be investigated that have a focus on the derivation of lightweight-driven PAs. This will form part of future research. To validate the three remaining requirements, all four designers were requested to rate the respective requirement on a scale from 0 ("unsatisfactory") to 4 ("very good") with regard to the method's application. The results revealed that the usefulness - here mainly efficiency - (average rating: 2.23) was considered too time-consuming. The main concern was the time needed to explain the various ratings for every DSM (mass-dependency schemes, see Figures 4-6). A more unified rating system (where possible) and quantitative measures might help with this point. This might also positively affect the comprehensibility (average rating: 2.75 ). By weighting the relevant lightweight motivations collectively within a team of designers (e.g., through pair comparison), the multiple rating steps might gain credibility, thus also improving the latter. Finally, the repeatability (averaged rating: 3.25 ) of the method results was rated as "good" by all designers.

\section{Conclusion}

The method presented in this paper provides a useful instrument for deriving lightweight-driven product architectures by redistributing the mass of components. By operationalizing the DSM, lightweight motivations can be applied to a decomposed system before the dependencies 
between the system elements are analyzed. A synthesis provides the designer with lightweightdriven modules and might support the novel arrangement of components or assemblies, thereby enabling the lack of methods for the design of a lightweight-driven product architecture to be addressed.

Further evaluation has yet to prove the flexibility and usefulness of the presented approach. The optimal application environment appears to fall between two extremes: products that are not too complex and justify investment of an appropriate amount of time into the method application, yet which are complex enough to be unmanageable without methodological support. Due to the results produced by the synthesis, methodological support is furthermore required downstream in the product-development process after application.

Nevertheless, the presented method appears to be useful for exploiting lightweight-design potential offered by the design of the product architecture. The intensification of the lightweight focus at an early point in the design process also helps to detect design trade-offs that might appear later on.

\section{Citations and References}

Ahn, H.-J., Lee, K.-H. \& Lee, C.-H. (2017). Design optimization of a knee joint for an active transfemoral prosthesis for weight reduction, Journal of Mechanical Science and Technology, Vol. 31, No. 12, pp. 5905-5913.

Browning, T. R. (2001). Applying the design structure matrix to system decomposition and integration problems: a review and new directions, Engineering Management, IEEE Transactions on, Vol. 48, No. 3, pp. 292-306.

Ericsson, A., \& Erixon, G. (1999). Controlling Design Variants: Modular Product-Platforms, Society of Manufacturing Engineers, Dearborn Michigan.

Gumpinger, T. (2016). Modulleichtbau - Methodische Unterstützung des Leichtbaus modularer Produktfamilien, PhD. Thesis Hamburg, Institute for Product Development and Mechanical Engineering Design, University Hamburg-Harburg, Vol. 11.

Hölttä-Otto, K., de Weck, O. (2007). Degree of Modularity in Engineering Systems and Products with Technical and Business Constraints, Concurrent Engineering: Research and Applications, Vol 15, No. 2, pp. 113-126.

Keller, A., \& Binz, H. (2009). Requirements on Engineering Design Methodologies, In Proceedings of the Int. Conf. on Engineering Design (ICED) 2009, Palo Alto, USA, pp. 317-329.

Laufer, F., Roth, D., \& Binz, H. (2019). An investigation into the influence of mass distribution on conceptual lightweight design, 29th CIRP Design Conference, Procedia CIRP 84 (2019), pp. 1041-1047.

Lehmann, J. F., Price, R., Okumura, R., Questad, K., de Lateur, B. J., \& Négretot, A. (1998). Mass and Mass Distribution of Below-Knee Prostheses: Effect oil Gait Efficacy and Self-Selected Walking Speed, Arch. Phys. Med. Rehabil., Vol. 79, pp. 162-168.

Lindemann, U., \& Maurer, M., \& Braun, T. (2009). Structural Complexity Management, Berlin, Heidelberg: Springer Verlag.

Luft, T., Klein, F., \& Wartzack, S. (2014). Weight and Cost Reduction of the Thermal Management for Traction Batteries by using the DSM Methodology, In Proceedings of DESIGN the 14th Int. Design Conf., in press, Dubrovnik, Croatia, pp. 293-302.

Mayyas, A., Omar, M., Hayajneh, M. \& Mayyas, A. R. (2017). Vehicle's lightweight design vs. Electrification from life cycle assessment perspective, Journal of Cleaner Production, Vol. 167, pp. 687-701.

McLellan, J., Maier, J. R. A., Fadel, G. M., \& Mocko, G. M. (2009). A Method for Identifying Requirements Critical to Mass Reduction using DSMS and DMMS, In Proceedings of 
11th International Design Structure Matrix Conference, DSM'09 Greenville, South Carolina, pp. 197-205.

Pimmler. T. U., \& Eppinger, S. D. (1994). Integration Analysis of Product Decompositions, ASME Conference on Design Theory and Methodology, Minneapolis, MN, September, pp. 343-351.

Posner, B. (2016). Methodik zum leichtbaugerechten Konzipieren, PhD. Thesis Stuttgart, Institute for Engineering Design and Industrial Design, University of Stuttgart, No. 656.

Richter, T., Inkermann, D., \& Vietor, T. (2016). Product architecture design as a key task within conceptual design, In Proceedings of DESIGN the 14th Int. Design Conf., in press, Dubrovnik, Croatia, pp. 1337-1346.

Roth, K. (2000). Konstruieren mit Konstruktionskatalogen - Konstruktionslehre, Vol. 1, 3. edition, Berlin, Heidelberg: Springer Verlag.

Steward, D. V. (1981). The Design structure system: A method for managing the design of complex systems, IEEE Transactions Engineering Management, vol. 28, pp. 71-74.

Torenbeek, E. (1976). Synthesis of subsonic airplane design, Delft University Press, Delft. https://doi.org/10.1007/978-94-017-3202-4

Trzesniowski, M. (2014). Rennwagentechnik, Springer Vieweg Verlag, Wiesbaden. https://doi.org/10.1007/978-3-658-04919-5

Wang, M., Beeh, E., Höfer, A., \& Friedrich H. (2018). Function Integration for Lightweight Chassis based on Axiomatic Design and Design Structure Matrix, International Journal of Automotive Technology, Vol. 19, No. 6, pp. 969-979.

Wojtkowiak, D., Malujda, I., Talaśka, K., Magdziak, Ł., \& Wieczorek, B. (2017). Influence of construction mass distribution on the walking robot's gait stability, 21st International Polish-Slovak Conference Machine Modeling and Simulations 2016, In Procedia Engineering 177, pp. 419-424. 